\section{Percepción de la señalización y orientación espacial de los usuarios de tres complejos hospitalarios de Santiago, Chile}

\author{
RODRIGO MORA ${ }^{1}$, AMALIA OATS $^{1}$, PÍA MARZIANO ${ }^{2}$
}

\section{A survey about client orientation and wayfinding in Chilean hospitals}

Background: Sense of orientation in hospitals can be tricky considering the large extension of buildings and the inadequate signage. Aim: To report some of the findings of a larger research project on wayfinding and patient navigation in Chilean hospitals. Material and Methods: Five hundred nine hospital users waiting for attention in three hospitals were contacted and asked to answer a survey that lasted 10 minutes, about wayfinding and sense of orientation within the hospital. Results: Users declared to have a good opinion of existing signage in the three hospitals analyzed as well as their architectural organization in terms of their capacity to orient people. However, the vast majority of users asked for directions to navigate within the hospital to staff and medical personnel. Conclusions: Patient navigation problems are imposing a great "hidden" cost to hospitals management due to missed appointments.

(Rev Med Chile 2014; 142: 1291-1296)

Key words: Hospitals; Orientation; Patient navigation.

\author{
'Escuela de Arquitectura, Facultad \\ de Arquitectura, Arte y Diseño, \\ Universidad Diego Portales. \\ ${ }^{2}$ Facultad de Arquitectura y \\ Urbanismo, Universidad de Chile. \\ Agradecimientos: Esta \\ investigacion contó con el apoyo \\ de Conicyt, bajo el proyecto \\ Fondecyt Iniciación Na11100020. \\ Recibido el 17 de diciembre de \\ 2013, aceptado el 27 de agosto \\ de 2014. \\ Correspondencia a: \\ RRodrigo Mora \\ Escuela de Arquitectura, Facultad \\ de Arquitectura, Arte y Diseño, \\ Universidad Diego Portales. \\ República 180, Santiago. \\ Teléfono: 226762702. \\ rodrigo.mora@udp.cl
}

E 1 conocer los procesos mentales y acciones que nos permiten orientarnos y llegar a nuestros destinos es la preocupación principal de la cognición espacial. En el mundo anglosajón existe una terminología específica para ambos procesos: "wayfinding"1-3, o proceso de toma de decisiones espaciales que permiten llegar desde un punto $\mathrm{A}$ a uno $\mathrm{B}$.

Uno de los espacios donde la orientación y el wayfinding tienen más relevancia son los hospitales, edificios donde se dan cita distintos grupos de personas (pacientes, visitantes, personal administrativo, y personal médico y paramédico), que se mueven en áreas bien delimitadas (pabellones quirúrgicos, oficinas de atención, habitaciones, etcétera), y donde en forma reiterada deben realizarse búsquedas de recintos específicos (por ejemplo, buscar la habitación de un pariente o la oficina de un cardiólogo).
Varias son las características que hace especialmente complejo orientarse y realizar wayfinding en hospitales. En primer lugar, existen problemas de terminología ${ }^{4}$, pues frecuentemente los términos usados por los usuarios no necesariamente coinciden con los usados por médicos (ej: rayos $\mathrm{X}$ versus imagenología). En segundo lugar, el proceso de crecimiento de los hospitales generalmente carece de un plan maestro que garantice la continuidad de circulaciones principales o prevea la localización de ascensores en situaciones futuras ${ }^{5,6}$, dificultando aunn más la orientación de los usuarios ${ }^{4}$. Finalmente, pacientes y visitantes suelen acudir en situaciones altamente estresantes (como por ejemplo, el nacimiento de un hijo), lo que reduce sus capacidades de concentración para tomar decisiones espaciales.

Los problemas de orientación en hospitales se traducen frecuentemente en pérdidas económicas 
Señalización y orientación en hospitales - R. Mora et al

importantes para estas instituciones, a la vez que un pobre servicio para los pacientes. Un estudio realizado por Ulrich y colegas ${ }^{8}$ en un hospital de casi 604 camas en Estados Unidos de Norteamérica mostró que se pierden cerca de 4,500 h de personal por año por la distracción que pacientes desorientados causan en personal administrativo y médico del hospital y la necesidad de reorientar a estos pacientes. Al año 2004 esto fue estimado en US\$202,000, lo que equivalía al costo de casi dos jornadas completas de un médico junior durante todo un año. Como parte de un proyecto de investigación reciente (Fondecyt Iniciación No 11100020), se demostró recientemente que los problemas de señalización al interior de un hospital chileno se traducen en que uno de cada seis personas camina al menos el doble de lo que debería haber caminado para llegar a sus destinaciones.

Se ha sostenido que la capacidad de "encontrar el camino" en forma efectiva en hospitales se traduce en una reducción del estrés y frustración en los visitantes y una mayor confianza en la labor hospitalaria ${ }^{9}$. Esta es quizás la razón por la cual se ha defendido la necesidad de un "diseño saludable" de los espacios hospitalarios, uno que tome en consideración, entre otras cosas, la planificación de la arquitectura y señalización que permitan la orientación de las personas ${ }^{10}$.

Con el objeto de avanzar en este objetivo, presentamos los resultados parciales de una investigación que buscó entender cómo los usuarios percibían y se orientaban en tres hospitales de tamaño mediano de Santiago. Los resultados presentados acá se refieren a la encuesta aplicada a más de 500 personas sobre la calidad de la señalización dispuesta en los hospitales, la arquitectura hospitalaria, y sobre las estrategias de orientación de las personas al interior de estos centros médicos.

\section{Los casos}

El primer caso corresponde al Hospital X, de aproximadamente $45,000 \mathrm{~m}^{2}$ y ubicado en un sector central de Santiago. Este hospital tiene cuatro accesos (dos por la calle al poniente y dos por la calle al oriente) y se organiza a la manera de torre, donde las unidades de hospitalización se desarrollan entre los pisos 2 y 8 . El segundo caso corresponde al Hospital Y, de $60,000 \mathrm{~m}^{2}$ aproximados y que, a diferencia del caso anterior, se organiza a partir de extensos corredores y patios y donde la mayoría de sus dependencias se encuentran en el primer piso. En este hospital existen una serie de huinchas de colores dispuestas en el piso para facilitar el acceso a las áreas más importantes del recinto. El último caso corresponde al Hospital Z, hospital privado asociado a la Facultad de Medicina de una de las principales universidades de Chile. Emplazado en el centro de la ciudad, este hospital de $26,000 \mathrm{~m}^{2}$ se estructura en dos partes, una al norte y otra al sur de una calle pública, las que se encuentran conectadas por un puente de uso interno sobre la calle. La Figura 1 muestra la planta del primer piso de los tres hospitales.

\section{Material y Método}

Para realizar la investigación se diseñó una encuesta que fue aplicada entre los meses de mayo de 2011 y mayo de 2012 a 509 personas aleatoriamente elegidas (16-83 años, promedio 41 años, $\mathrm{DE}=14,2)$, de los tres hospitales (Tabla 1). La encuesta contenía diez preguntas en total y fue diseñada por el investigador responsable de esta investigación en base a las guías de diseño inglesas para la evaluación de sistemas de señalizacion en hospitales ${ }^{11}$.

Tabla 1.

\begin{tabular}{|lccccccccccc|}
\hline & Hombres & $\begin{array}{l}\text { Sexo } \\
\text { Mujeres }\end{array}$ & Total & $\begin{array}{c}\text { Familiaridad } \\
\text { Personal } \\
\text { médico }\end{array}$ & $\begin{array}{c}\text { Pacien- } \\
\text { tes }\end{array}$ & $\mathbf{1 6 - 3 0}$ & $\begin{array}{c}\text { Edad } \\
\mathbf{3 1 - 5 0}\end{array}$ & $\begin{array}{c}\mathbf{5 1} \mathbf{y} \\
\text { más }\end{array}$ & $\begin{array}{c}\text { Nivel educacional } \\
\text { Ens. } \\
\text { Básica }\end{array} \begin{array}{c}\text { Ens. } \\
\text { Media Superior } \\
\text { Ens. }\end{array}$ \\
Hospital X & 58 & 82 & 140 & 38 & 102 & 24 & 97 & 19 & 39 & 83 & 18 \\
Hospital Y & 56 & 144 & 200 & 0 & 200 & 51 & 61 & 88 & 27 & 82 & 91 \\
Hospital Z & 65 & 104 & 169 & 30 & 139 & 51 & 67 & 51 & 128 & 41 & 0 \\
\hline
\end{tabular}




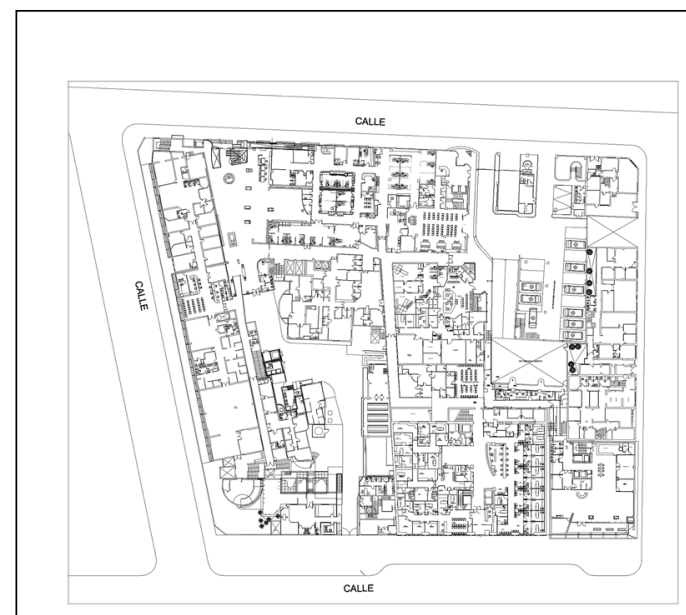

Hospital X
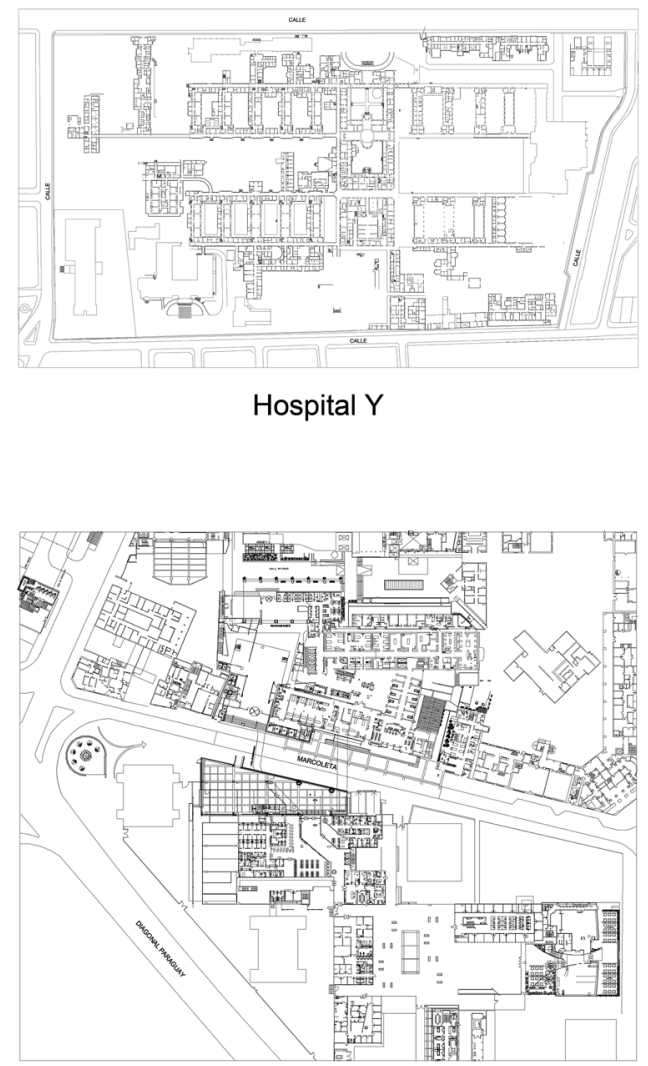

Hospital Z

Figura 1. Planta de primer piso de los hospitales $X, Y$ y $Z$. Fuente: Elaboración propia.
Con el fin de validar las preguntas y asegurar que fueran comprensibles para los encuestados se realizó un pre-test a 10 personas aleatoriamente elegidas en marzo de 2010. Los resultados de este pre-test permitieron definir el cuestionario final, que fue aplicado luego por un encuestador capacitado. Para la realización de la encuesta se contó con la autorización escrita de cada uno de los hospitales. Asimismo, los pacientes firmaron un consentimiento informado (previamente visado por el comité de ética de la Universidad de Santiago y aprobado por Conicyt), antes de responder el cuestionario.

\section{Tamaño de la muestra}

Dado que no es posible conocer la población real de usuarios de cada hospital, se asume que la población es infinita y con varianza máxima. Asumiento una selección aleatoria simple de observaciones, se obtiene un nivel de error de $4,3 \%$, con un intervalo de confianza de $95 \%$.

Los aspectos analizados por la encuesta fueron tres:

Valoración de capacidad de orientación. Primeramente, se preguntó por una auto-evaluación de la capacidad de orientación en el espacio hospitalario (rango 1 a 7 , donde 1 es muy pobre orientación y 7 muy buena orientación), y en términos generales (mismo rango).

Valoración de la señalización. En segundo lugar se pidió a los usuarios que evaluaran, en forma separada, la señalización presente en el hospital en forma separada en términos de su cantidad, ubicación y calidad, así como la capacidad de la organización arquitectónica de cada hospital en facilitar o dificultar la orientación. Se usaron para ello cinco categorías: muy fácil, fácil, neutro (ni fácil ni dificil), difícil y muy difícil.

Identificación de estrategias de orientación. Se buscó conocer las estrategias usadas por las personas para orientarse en el hospital. Para ello se preguntó a las personas qué es lo primero que hacían para orientarse en el hospital: preguntar a guardias o personal administrativo, preguntar a médicos o personal del hospital, consultar mapas, leer señalización y finalmente usar otra estrategia. 


\section{Análisis estadístico}

Se aplicaron las pruebas estadísticas $\chi^{2}$ y prueba $\mathrm{T}$, dependiendo de la naturaleza de la variable dependiente. Se buscó determinar relaciones entre variables de género, edad y nivel educacional, grado de familiaridad con el hospital y las respuestas de los individuos en las dimensiones mencionadas en el punto anterior. Se agrupó la edad de los participantes en tres grupos: de 16 a 29 (jóvenes y adultos jóvenes), de 30 a 49 (adultos) y de 50 y más y se los sometió a un análisis de varianza (ANOVA). Se consideró estadísticamente significativo un $\mathrm{p}<0,05$.

\section{Resultados}

El 35\% (179) de los encuestados fueron hombres. El 86,6\% $(\mathrm{n}=441)$ correspondieron a pacientes, $3,5 \%(\mathrm{n}=18)$ médicos y paramédicos y $9,8 \%(\mathrm{n}=50)$, a personal administrativo. El $38,1 \%(n=194)$ de los encuestados declaró tener estudios superiores o técnicos, 40,5\% $(\mathrm{n}=206)$ sólo haber completado la enseñanza media y 21,4\% (109), sólo haber cursado la educación básica (completa o incompleta). El 22\% $(\mathrm{n}=96)$ declaró haber visitado el hospital por primera vez o sólo una vez antes, mientras 78,8\% $(\mathrm{n}=344)$ dijo haber estado en los recintos hospitalarios anteriormente más de dos veces (68 personas, o $13,4 \%$, no respondieron).

Respecto de la capacidad de orientación en el espacio hospitalario, se encontró que en promedio los encuestados se auto calificaban con 5,62 $(\mathrm{DE}=1,38)$ en una escala del 1 al 7 , y que sólo $7,5 \%$ de ellos $(\mathrm{n}=38)$, se ponían una nota menor a 4. En lo que refiere a la capacidad de orientación general, el promedio de los encuestados se auto calificó con 6,09 $(\mathrm{DE}=1,14)$, mientras que sólo $3,4 \%(\mathrm{n}=17)$ de los encuestados con una calificación menor a 4 .

Los encuestados valoraron positivamente la cantidad de señalización (promedio 5,69 $(\mathrm{DE}=1,34)$ ), la ubicación de la señalización (promedio 5,61 (DE = 1,4)) y claridad de la señalización (promedio 5,62 (DE = 1,34)). La evaluación de la organización arquitectónica en relación a la capacidad de orientar a los visitantes fue peor evaluada, pues $36 \%$ encontró que esta hacía fácil la orientación, mientras $36 \%$ que lo hacía difícil o muy difícil. El $28 \%$ consideró que la arquitectura no hacía ni fácil ni difícil a los usuarios orien- tarse en los hospitales. La alta valoración de la señalización no se tradujo necesariamente en su uso. El $67,6 \%$ de los encuestados $(\mathrm{n}=344)$ dijo que la primera estrategia para orientarse era la de preguntar a personal administrativo (guardias, recepcionistas o personal de aseo) o médico, mientras que $20,3 \%$ dijo que leía señalización o mapas. El 11\% restante dijo usar otra estrategia.

No se detectaron diferencias entre hombres y mujeres sobre su capacidad de orientación en los hospitales (promedio hombres 5,61 $(\mathrm{n}=179)$, y mujeres 5,63 $(\mathrm{n}=330), t(509)=-0,143, \mathrm{p}=0,88)$. Aunque los hombres declararon tener una mejor auto evaluación de sí mismos que las mujeres $(6,19(\mathrm{DE}=1,02)$ versus $6,03(\mathrm{DE}=1,19)$, $\mathrm{t}(509)=-1,49, \mathrm{p}=0,13)$, las mujeres evaluaron a la arquitectura de mejor forma que los hombres $(5,68(\mathrm{DE}=1,28) \mathrm{t}(509)=-0,16, \mathrm{p}=0,86)$ versus $5,39(\mathrm{DE}=1,02)$.

No se encontró diferencias estadísticamente significativas entre grupos en lo que refiere a capacidad de orientación en hospitales, capacidad de orientación a nivel general, percepción sobre la cantidad, ubicación y claridad de la señalización y percepción de la arquitectura hospitalaria. Sin embargo, sí se detectaron diferencias estadísticamente significativas por hospital en lo que respecta a la capacidad de orientarse en ellos (hospital X 5,96 comparado con 5,51 y 5,47 de los hospitales Y y Z respectivamente, $\mathrm{F}(3,509)$ ), cantidad de señalización (hospital X 6,2 comparado con 5,47 y 5,53 de hospitales $\mathrm{Y}$ y Z respectivamente, $\mathrm{F}(3,509)=15,4$ $\mathrm{p}=0,01$ ), ubicación de señalización (hospital $\mathrm{X}$ 6,14 comparado con 5,39 y 5,46 de hospitales $Y$ y $\mathrm{Z}$ respectivamente, $\mathrm{F}(3,509)=14,2 \mathrm{p}=0,01), \mathrm{y}$ claridad de esta (hospital X 6,01 comparado con 5,84 y 516 de hospitales $\mathrm{Y}$ y $\mathrm{Z}$ respectivamente, $\mathrm{F}$ $(3,509)=8,9 \mathrm{p}=0,01)$.

Se detectó además que el nivel educacional afectaba la evaluación de la señalización. Un análisis de varianza (ANOVA) mostró que las personas que sólo habían completado parcial o totalmente la educación básica, confiaban menos en su capacidad de orientación general que los que tenían educación media o superior $(5,79$ contra 6,23 y $6,09$ respectivamente, $\mathrm{F}(3,509)=5,3 \mathrm{p}=0,005)$, que evaluaban con peor nota la cantidad de señalización (5,32 contra 5,84 y 5,74 respectivamente, $\mathrm{F}(3,509)=5,82 \mathrm{p}=0,003)$, la ubicación de la señalización $(5,26$ contra 5,78 y 5,62 respectivamente, $\mathrm{F}(3,509)=5,06 \mathrm{p}=0,007)$, y la claridad 
de la señalización $(5,31$ contra 5,73 y 5,68 respectivamente, , $\mathrm{F}(3,509)=3,79 \mathrm{p}=0,024)$.

Con respecto a las estrategias usadas por las personas para orientarse se diferenció entre pacientes y familiares de pacientes, por una parte, $\mathrm{y}$ funcionarios del hospital (guardias, recepcionistas, personal médico y para-médico, personal administrativo), por otra. Estos dos grupos fueron comparados con tres tipos de estrategias de orientación; consulta de mapas y de señalización, consulta verbal a personal del hospital (guardias, recepcionistas, administrativos y médicos), y otras estrategias. Los resultados no mostraron diferencias estadísticamente significativas de orientación entre grupos: mientras en el primer caso el $66,2 \%$ pregunta a otros la localización de áreas no conocidas, en el caso de los visitantes este porcentaje alcanza el $67,8 \%\left(\chi^{2}(2, n=509)=, 462\right.$, $\mathrm{p}<, 01)$. Tampoco se detectaron diferencias en las estrategias de orientación de hombres y mujeres $\left(\chi^{2}(2, \mathrm{n}=509)=2,16, \mathrm{p}<, 01\right)$, por cuanto tanto los primeros como las segundas recurren mayoritariamente a información provista por personal del hospital (médicos, recepcionistas y personal administrativo), por sobre la consulta de la señalización y mapas. Finalmente, no se detectaron diferencias estadísticamente significativas entre el nivel educacional y las estrategias para orientarse. $\left(\left(\chi^{2}(3, \mathrm{n}=509)=10,18, \mathrm{p}<, 01\right)\right.$.

\section{Discusión}

Los resultados presentados sugieren que al margen de las diferencias en la arquitectura de cada uno de los hospitales y su capacidad de orientar a los ususarios (aspecto detectado en esta investigación), existen ciertos aspectos comunes en la forma en que los pacientes perciben la señalización y la organización arquitectónica de los recintos, así como en las estrategias usadas para moverse en ellos. Esto porque el comportamiento "por defecto" de los visitantes de hospitales analizados es el de preguntar a personal médico y administrativo por la localización de recintos, más que observar la señalización dispuesta para orientarlos.

Lo anterior sugiere la existencia de una característica cultural arraigada, la omnipresencia de la oralidad por sobre el mensaje escrito. Lo anterior parece contrastar con lo reportado en estudios de este tipo realizados en países desarrollados ${ }^{13,14}$, que han mostrado que usuarios de edificios com- plejos usan no una sino múltiples estrategias para orientarse en edificios complejos, dentro de las cuales está el preguntar por instrucciones verbales a terceros.

Hölscher y otros ${ }^{14}$, por ejemplo, en su estudio de un edificio multinivel complejo, mostró que las personas con menor familiaridad con un edificio tenían como estrategia acceder a los lugares centrales de un edificio para luego dirigirse a cada una de las alas, mientras que las personas con mayor grado de familiaridad buscaban primer el nivel al cual debían ir para luego dirigirse a los sectores de destino. Estas estrategias no sólo operaban a un nivel macro, sino afectaban también la forma en que las personas se movían al interior de los edificios, pues mientras las personas con mayor nivel de familiaridad con un sistema espacial se movían por los espacios principales conservando lo más posible trayectorias rectas ${ }^{15}$, las con menor nivel de conocimiento del edificio buscaban reducir la distancia métrica de sus rutas a través de encontrar atajos, lo que las llevaba a complejizar las rutas elegidas. En este sentido, un grado de familiaridad con el edificio lleva a que el proceso de exploración y búsqueda de destinaciones pase de ser concebido por las personas como un plan de tipo general, con acciones y etapas, a un proceso más precario definido paso a paso y de forma contextual ${ }^{16}$.

La importancia de la información verbal para la orientación de las personas en hospitales chilenos plantea tres grandes preguntas. La primera es sobre qué tipo de información esta siendo transmitida a los usuarios y quién o quienes la estan transmitiendo. Se mencionó anteriormente que un problema frecuente de los hospitales es que la señalización contiene información que no es fácil de entender para las personas: el término imagenología, popularmente conocido como rayos X es un buen ejemplo.

Otro aspecto de interés es quienes están transmitiendo la información sobre orientación en los hospitales. El hecho de que la mayoría de las personas recurra a guardias y personal administrativo no sólo plantea la pregunta de cuántas horas laborales están siendo ocupadas en tareas no consideradas en estos cargos sino además sobre la capacidad real que estos funcionarios tienen para orientar a terceros. Puede ser el caso que la información trasmitida no sea clara o incluso induzca a más errores de orientación. Estudios recientes ${ }^{12}$ sugieren que, al momento de entregar informa- 
Señalización y orientación en hospitales - R. Mora et al

ción sobre una posible ruta, las personas tienden a complejizar o simplificar la instrucción según la persona que esta recibiendo tal información. Cuando es un tercero el que recibe la información las instrucciones tienden a simplificarse, lo que se traduce en que las trayectorias resultantes de tales instrucciones no sean necesariamente "óptimas" desde el punto de vista de su distancia, pero que tengan menos cambios de dirección. Cuando en cambio es uno mismo el potencial receptor del mensaje, la instrucción suele ser más "eficiente" (lease corta) desde el punto de vista de su largo, pero al mismo tiempo más compleja en términos de cambios de dirección. La inexistencia de estudios que profundicen en lo anterior en el contexto chileno hace que la pertinencia de estas ideas a nuestra realidad sea por ahora sólo materia de especulación.

La segunda pregunta se refiere al rol de la señalización en los hospitales. A pesar de que existe una buena evaluación de ella (a juzgar por los resultados de esta investigación), las personas mayoritariamente no usan esta información para moverse en hospitales sino que prefieren preguntarle a terceros cómo llegar a diferentes destinos. Esto, además de sugerir que la señalización no estaría cumpliendo el rol para el cual fue confeccionada, lleva a preguntarse cuál es el impacto económico real que los problemas de orientación de los usuarios están causando en los hospitales chilenos. Según uno de los directores médicos de los hospitales analizados en esta investigación, varias horas médicas de un escáner de última generación recientemente adquirido se pierden al mes porque los usuarios llegan tarde a sus citaciones debido a que se desorientan. Visto así, es posible que una parte de los (históricamente insuficientes) dineros públicos destinados a los hospitales, se esté perdiendo por causa de problemas de navegación de los usuarios, un contrasentido de proporciones en cualquier política de salud.

\section{Referencias}

1. Arthur P, Passini R. Wayfinding-People, Signs and Architecture. McGraw Hill Ryerson Limited, 1992

2. Carpman JR, Grant MA. Healthcare Design. Marberry,
S.O., Wileys NY, 1996.

3. Haq S, Zimring C. Just down the road a piece. The development of topological knowledge of buildings layouts. Environment and Behavior 2006; 35: 132-60.

4. Cooper R. Wayfinding for healthcare. Aha Press, Chicago, 2010

5. Peponis J, Zimring C, Kuing Choi Y. Finding the building in wayfinding Environment \& Behaviorm 1990; 22: 555 90.

6. O’Neill MJ. Effects on Signage and Floorplan Configuration on Wayfinding Accuracy. Environment and Behavior 1991; 23: 553-74.

7. Omer I, Goldblatt GR. The implications of inter-visibility between landmarks on wayfinding performance: An investigation using a virtual urban environment. Computers, Environment and Urban Systems 2007; 31: 520-34.

8. Ulrich R, Quan X, Zimring C, Joseph A, Choudhary $\mathrm{R}$. The Role of the Physical Environment in the Hospital of the 21st Century: A Once-in a-Lifetime Opportunity. Georgia Institute of Technology, 2004.

9. Passini R. Proceedings of the International Conference on Building Use and Safety Technology, Signs systems, Maps and Wayfinding, 1985: 35-41. National Institute of Building Sciences, Washington D.C., USA.

10. McCarthy M. Healthy Design. The Lancet 2004; 364: 405-6.

11. Wayfinding. Effective wayfinding ans signing systems, guidance for healthcare facilities, NHS Estates, 1999. Acceso, Noviembre 12, 2014. Disponible en: https:// www.gov.uk/government/uploads/system/uploads/ attachment_data/file/148500/Wayfinding.pdf

12. Lawton C. Strategies for indoor wayfinding: the role of orientation. Journal of Environmental Psychology 16; 2 : 137-45.

13. Hölscher Ch, Tenbrik T, Wiener J. Would you follow your own route description? Cognitive strategies and route planning. Cognition 2011; 121: 228-47.

14. Hölscher Ch, Meilinger T, Vrachliotisa G, Brösamle M, Knauff M. Up the down staircase: Wayfinding strategies in multi-level buildings. Journal of Environmental Psychology 2006; 26: 284-99.

15. Conroy-Dalton R. The secret is to follow your nose. Environment and Behavior 2003; 35: 107-31.

16. Kato $Y$, Takeuchi Y. Individual differences in wayfinding strategies. Journal of Environmental Psychology 2003; 23: $171-88$. 